\title{
Eigenvalue Problems for Fractional $p(x, y)$-Laplacian Equations with Indefinite Weight
}

\author{
Nguyen Thanh Chung
}

\begin{abstract}
In this paper, we consider a class of eigenvalue problems for fractional $p(x, y)$-Laplacian equations with indefinite weight in fractional Sobolev space with variable exponent. Under some suitable conditions on the growth rates involved in the problem, we establish some results on the existence of a continuous family of eigenvalues using variational techniques and Ekeland's variational principle.
\end{abstract}

\section{Introduction}

In this paper, we are interested in a class of eigenvalue problems for the following fractional $p(x, y)$-Laplacian equation

$$
\begin{cases}\mathcal{L}_{p(x, y)} u+|u|^{q(x)-2} u=\lambda V(x)|u|^{r(x)-2} u, & x \in \Omega \\ u=0, & x \in \partial \Omega\end{cases}
$$

where $\Omega \subset \mathbb{R}^{N}, N \geq 2$ is a bounded domain with Lipschitz boundary, $p: \bar{\Omega} \times \bar{\Omega} \rightarrow \mathbb{R}$ is continuous, $q, r \in C_{+}(\bar{\Omega}), V: \Omega \rightarrow \mathbb{R}$ is an indefinite weight function in the sense that it is allowed to change sign in $\Omega, \lambda$ is a positive constant and $\mathcal{L}_{p(x, y)}(\cdot)$ is the fractional $p(x, y)$-Laplace operator, i.e.,

$$
\mathcal{L}_{p(x, y)} u:=\text { p.v. } \int_{\Omega} \frac{|u(x)-u(y)|^{p(x, y)-2}(u(x)-u(y))}{|x-y|^{N+s p(x, y)}} d y, \quad s \in(0,1) .
$$

Throughout this paper, we assume that

$$
p(x, x)<q(x)<p_{s}^{*}(x):=\frac{N p(x, x)}{N-s p(x, x)}, \quad p(x, y)=p(y, x)<\frac{N}{s}, \quad \forall x, y \in \bar{\Omega},
$$

where $p_{s}^{*}(x)$ is the so-called critical exponent in fractional Sobolev space with variable exponent. In the constant exponent case, the operator $\mathcal{L}_{p(x, y)}$ defined above is known as the fractional $p$-Laplacian. On the other hand, we remark that it is a fractional version of the well known $p(x)$-Laplacian. Recently, great attention has been focused on elliptic

Received September 6, 2018; Accepted April 9, 2019.

Communicated by Jann-Long Chern.

2010 Mathematics Subject Classification. 35B60, 35J20, 35J91, $46 \mathrm{E} 35$.

Key words and phrases. fractional $p(x, y)$-Laplacian problems, indefinite weight, fractional Sobolev space, variable exponent, variational methods. 
equations involving fractional operators, both for pure mathematical research and in view of concrete real-world applications. This type of operator arises in a quite natural way in many different contexts, such as, among others, the thin obstacle problem, optimization, finance, phase transitions, soft thin films, conservation laws, multiple scattering, minimal surfaces, material science, .... The fractional Laplacian also provides a simple model to describe certain jump Lévy processes in probability theory. The cited results turn out to be very fruitful in order to recover an elliptic PDE approach in a fractional framework, we refer to some interesting papers [5, 11, 12, 18, 25, 28 and the monograph of Bisci et al. [13] for a thorough variational approach of fractional Sobolev spaces and corresponding nonlocal problems.

In recent years, the study of differential equations and variational problems involving variable exponent conditions has been an interesting topic. The interest in studying such problems was stimulated by their applications in elastic mechanics, fluid dynamics and the mathematical models of stationary thermo-rheological viscous flows of non-Newtonian fluids. For more information on modeling physical phenomena by equations involving $p(x)$-growth condition we refer to [2,27]. We know that the $p(x)$-Laplacian operator where $p(\cdot)$ is a continuous function possesses more complicated properties than the $p$ Laplacian operator, mainly due to the fact that it is not homogeneous. There has been many works devoted to the existence of solutions for variable exponent problems, both on bounded domain and unbounded domain, we refer to [1,3, 10, 14, 16, 20, 22, 26]. In [4, 9], the authors considered the existence, nonexistence and properties of solutions for $p(x)$ curl systems or $p(x, t)$-curl systems arising in electromagnetism. A natural question to see what results can be recovered when the $p(x)$-Laplace operator is replaced by the fractional $p(x, y)$-Laplacian of the form $\mathcal{L}_{p(x, y)}$. To our best knowledge, Kaufmann et al. 19 and Pezzo et al. 24 firstly introduced some results on fractional Sobolev spaces with variable exponent $W^{s, q(x), p(x, y)}(\Omega)$ and the fractional $p(x, y)$-Laplacian. There, the authors established compact embedding theorems of these spaces into variable exponent Lebesgue spaces. As an application, they also prove an existence result for nonlocal problems involving the fractional $p(x, y)$-Laplacian. In [8], Bahrouni et al. obtained some further qualitative properties of the fractional Sobolev space $W^{s, q(x), p(x, y)}(\Omega)$ and the fractional $p(x, y)$-Laplacian $\mathcal{L}_{p(x, y)}$. After that, some studies on this kind of problems are performed by using different approaches, see [6, 7, 29.

In this paper, we are motivated by the results on the $p(x)$-Laplacian problems with weight introduced in [1, 14,20,22] and some results on the theory of fractional Sobolev spaces with variable exponent due to Kaufmann et al. [19] and Bahrouni et al. [8]. In [21], Mihailescu et al. studied an eigenvalue problem with non-negative weight for the Laplace operator on a bounded domain with smooth boundary in $\mathbb{R}^{N}, N \geq 3$. They showed the 
existence of two positive constants $\lambda_{*}$ and $\lambda^{*}$ with $\lambda_{*} \leq \lambda^{*}$ such that any $\lambda \in\left(0, \lambda_{*}\right)$ is not an eigenvalue of the problem while any $\lambda \in\left[\lambda^{*},+\infty\right)$ is an eigenvalue of the problem. Some similar results for a class of $p(x)$-Laplacian problems involving multiple variable exponents can be found in [22], in which the authors considered the problem with indefinite weight. We also refer to [1,14,20 for some further studies on the topic. Our aim is to study a class of eigenvalue problems with indefinite weight for fractional $p(x, y)$-Laplacian equations. The situation here is different from those performed in the previous papers in the sense that we deal with problem (1.1) in a fractional framework. We also consider problem (1.1) in both cases the nonlinear term is sublinear or superlinear at infinity. Finally, it should be noticed that the obtained results here also complement and generalize Theorem 5.1 of [8] and Theorem 1.4 of [19]. The main tools which will be used in this paper comes from some variational techniques and Ekeland's variational principle [17.

\section{Preliminaries}

We recall, in what follows, some definitions and basic facts about the setup for generalized Lebesgue spaces $L^{h(x)}(\Omega)$ and fractional Sobolev spaces with variable exponent $W_{0}^{s, q(x), p(x, y)}(\Omega)$, where $\Omega$ is a bounded domain in $\mathbb{R}^{N}$, with a smooth boundary $\partial \Omega$. In that context we refer to the books of Diening et al. 15] and Musielak 23, the papers of Edmunds et al. [16], Bahrouni et al. [8], Kaufmann et al. [19] and their references. Set

$$
C_{+}(\bar{\Omega})=\{h ; h \in C(\bar{\Omega}), h(x)>1 \text { for any } x \in \bar{\Omega}\} .
$$

For $h(x) \in C_{+}(\bar{\Omega})$, we define the variable exponent Lebesgue space $L^{h(x)}(\Omega)$ by

$$
L^{h(x)}(\Omega)=\left\{u ; u \text { is a measurable real-valued function, } \int_{\Omega}|u(x)|^{h(x)} d x<\infty\right\} .
$$

The Luxemburg norm on this space is given by the formula

$$
|u|_{h(x)}:=\inf \left\{\mu>0, \int_{\Omega}\left|\frac{u(x)}{\mu}\right|^{h(x)} d x \leq 1\right\} .
$$

It's well known, that $\left(L^{h(x)}(\Omega) ;|\cdot|_{h(x)}\right)$ is a separable, uniformly convex Banach space. $\left(L^{h(x)}(\Omega) ;|\cdot|_{h(x)}\right)$ is called a generalized Lebesgue space. Moreover, its conjugate space is $L^{h^{\prime}(x)}(\Omega)$, where $1 / h^{\prime}(x)+1 / h(x)=1$. For $u \in L^{h(x)}(\Omega)$ and $v \in L^{h^{\prime}(x)}(\Omega)$, one has the following Hölder type inequality

$$
\left|\int_{\Omega} u(x) v(x) d x\right| \leq\left(\frac{1}{h^{-}}+\frac{1}{h^{\prime-}}\right)|u|_{h(x)}|v|_{h^{\prime}(x)} \leq 2|u|_{h(x)}|v|_{h^{\prime}(x)},
$$

where $h^{-}:=\min _{\bar{\Omega}} h(x)$ and $h^{\prime-}:=\min _{\bar{\Omega}} h^{\prime}(x)$. Moreover, if $h_{1}, h_{2}, h_{3} \in C_{+}(\bar{\Omega})$ and $1 / h_{1}(x)+1 / h_{2}(x)+1 / h_{3}(x)=1$, then for any $u \in L^{h_{1}(x)}(\Omega), v \in L^{h_{2}(x)}(\Omega)$ and $w \in$ 
$L^{h_{3}(x)}(\Omega)$ we have

$$
\left|\int_{\Omega} u v w d x\right| \leq\left(\frac{1}{h_{1}^{-}}+\frac{1}{h_{2}^{-}}+\frac{1}{h_{3}^{-}}\right)|u|_{h_{1}(x)}|v|_{h_{2}(x)}|w|_{h_{3}(x)} .
$$

Note that $L^{h_{2}(x)}(\Omega) \hookrightarrow L^{h_{1}(x)}(\Omega)$ for all functions $h_{1}$ and $h_{2}$ in $C_{+}(\bar{\Omega})$ satisfying $h_{1}(x) \leq h_{2}(x)$ for any $x \in \bar{\Omega}$. In addition this embedding is continuous.

An important role in manipulating the generalized Lebesgue spaces is played by the modular of the $L^{h(x)}(\Omega)$ space, which is the mapping $\rho_{h(x)}: L^{h(x)}(\Omega) \rightarrow \mathbb{R}$ defined by

$$
\rho_{h(x)}(u)=\int_{\Omega}|u|^{h(x)} d x
$$

If $\left(u_{n}\right), u \in L^{h(x)}(\Omega)$ and $h^{+}:=\max _{x \in \bar{\Omega}} h(x)<+\infty$, then the following relations hold true

$$
\begin{gathered}
|u|_{h(x)}>1 \quad \Longrightarrow \quad|u|_{h(x)}^{h^{-}} \leq \rho_{h(x)}(u) \leq|u|_{h(x)}^{h^{+}}, \\
|u|_{h(x)}<1 \quad \Longrightarrow \quad|u|_{h(x)}^{h^{+}} \leq \rho_{h(x)}(u) \leq|u|_{h(x)}^{h^{-}}, \\
\left|u_{n}-u\right|_{h(x)} \rightarrow 0 \quad \text { if and only if } \rho_{h(x)}\left(u_{n}-u\right) \rightarrow 0 .
\end{gathered}
$$

Proposition 2.1. (see [16]) Let $h_{1}$ and $h_{2}$ be measurable functions such that $h_{1} \in L^{\infty}\left(\mathbb{R}^{N}\right)$ and $1 \leq h_{1}(x) h_{2}(x) \leq+\infty$ for a.e. $x \in \mathbb{R}^{N}$. Let $u \in L^{h_{2}(x)}\left(\mathbb{R}^{N}\right), u \neq 0$. Then we have the following assertions

$$
\begin{aligned}
|u|_{h_{1}(x) h_{2}(x)} \leq 1 & \Longrightarrow \quad|u|_{h_{1}(x) h_{2}(x)}^{h_{1}^{+}} \leq\left.\left.|| u\right|^{h_{1}(x)}\right|_{h_{2}(x)} \leq|u|_{h_{1}(x) h_{2}(x)}^{h_{1}^{-}}, \\
|u|_{h_{1}(x) h_{2}(x)} \geq 1 & \Longrightarrow \quad|u|_{h_{1}(x) h_{2}(x)}^{h_{1}^{-}} \leq\left.\left.|| u\right|^{h_{1}(x)}\right|_{h_{2}(x)} \leq|u|_{h_{1}(x) h_{2}(x)}^{h_{1}^{+}}
\end{aligned}
$$

In particular, if $h_{1}(x)=h_{1}$ is a constant, then it holds that

$$
\left.\left.|| u\right|^{h_{1}}\right|_{h_{2}(x)}=|u|_{h_{1} h_{2}(x)}^{h_{1}} .
$$

For the convenience of the readers, we recall some definitions and basic properties of variable exponent Sobolev fractional spaces. For a deeper treatment on these spaces, we refer to $8,19,24]$.

For a smooth bounded domain $\Omega$ in $\mathbb{R}^{N}$, we consider two continuous functions $p: \bar{\Omega} \times$ $\bar{\Omega} \rightarrow(1,+\infty)$ and $q: \bar{\Omega} \rightarrow \mathbb{R}$. We assume that $p$ is symmetric, i.e., $p(x, y)=p(y, x)$ for all $x, y \in \bar{\Omega}$ and

$$
\begin{gathered}
1<p^{-}:=\min _{(x, y) \in \bar{\Omega} \times \bar{\Omega}} p(x, y) \leq p(x, y) \leq p^{+}:=\max _{(x, y) \in \bar{\Omega} \times \bar{\Omega}} p(x, y)<+\infty \\
1<q^{-}:=\min _{x \in \bar{\Omega}} q(x) \leq q(x) \leq q^{+}:=\max _{x \in \bar{\Omega}} q(x)<+\infty
\end{gathered}
$$


For $s \in(0,1)$, the fractional Sobolev space with variable exponent via the Gagliardo approach $X=W^{s, q(x), p(x, y)}(\Omega)$ is defined as follows

$$
X=\left\{u \in L^{q(x)}(\Omega), \int_{\Omega \times \Omega} \frac{|u(x)-u(y)|^{p(x, y)}}{\mu^{p(x, y)}|x-y|^{N+s p(x, y)}} d x d y<+\infty \text { for some } \mu>0\right\} .
$$

Let

$$
[u]_{s, p(x, y)}:=\inf \left\{\mu>0, \int_{\Omega \times \Omega} \frac{|u(x)-u(y)|^{p(x, y)}}{\mu^{p(x, y)}|x-y|^{N+s p(x, y)}} d x d y<1\right\}
$$

be the variable exponent Gagliardo seminorm and define

$$
\|u\|_{X}=[u]_{s, p(x, y)}+|u|_{q(x)} .
$$

Then $X$ equipped with the norm $\|\cdot\|_{X}$ is a Banach space. For $u \in X$, we set

$$
\rho(u)=\int_{\Omega \times \Omega} \frac{|u(x)-u(y)|^{p(x, y)}}{|x-y|^{N+s p(x, y)}} d x d y+\int_{\Omega}|u|^{q(x)} d x
$$

and

$$
\|u\|_{\rho}=\inf \left\{\mu>0, \rho\left(\frac{u}{\mu}\right) \leq 1\right\} .
$$

It's well known that $\|\cdot\|_{\rho}$ is a norm which is equivalent to the norm $\|\cdot\|_{X}$. Due to Lemma 3.1 in [19], $\left(X,\|\cdot\|_{X}\right)$ is a separable and reflexive Banach space.

To take into account the boundary condition in problem 1.1) we consider the space $X_{0}=W_{0}^{s, q(x), p(x, y)}(\Omega)$ that is the closure of $C_{0}^{\infty}(\Omega)$ in $X$. Then $X_{0}$ is a separable and reflexive Banach space under the norm

$$
\|u\|=[u]_{s, p(x, y)} .
$$

Proposition 2.2. $\quad$ (i) If $1 \leq[u]_{s, p(x, y)}<+\infty$, then

$$
\left([u]_{s, p(x, y)}\right)^{p^{-}} \leq \int_{\Omega \times \Omega} \frac{|u(x)-u(y)|^{p(x, y)}}{|x-y|^{N+s p(x, y)}} d x d y \leq\left([u]_{s, p(x, y)}\right)^{p^{+}} .
$$

(ii) If $[u]_{s, p(x, y)} \leq 1$, then

$$
\left([u]_{s, p(x, y)}\right)^{p^{+}} \leq \int_{\Omega \times \Omega} \frac{|u(x)-u(y)|^{p(x, y)}}{|x-y|^{N+s p(x, y)}} d x d y \leq\left([u]_{s, p(x, y)}\right)^{p^{-}} .
$$

In the following lemma, we give a compact embedding result into the variable exponent Lebesgue spaces. 
Proposition 2.3. (see [19]) Let $\Omega \subset \mathbb{R}^{N}$ be a Lipschitz bounded domain and $s \in(0,1)$. Let $q(x), p(x, y)$ be continuous variable exponents with $s p(x, y)<N$ for all $(x, y) \in \bar{\Omega} \times \bar{\Omega}$ and $q(x)>p(x, x)$ for all $x \in \bar{\Omega}$. Assume that $r: \bar{\Omega} \rightarrow(1,+\infty)$ is a continuous function such that

$$
p_{s}^{*}(x):=\frac{N p(x, x)}{N-s p(x, x)}>r(x) \geq r^{-}>1
$$

for all $x \in \bar{\Omega}$. Then, there exists a constant $c=c(N, s, p, q, r, \Omega)$ such that for every $u \in X=W^{s, q(x), p(x, y)}(\Omega)$, it holds that

$$
|u|_{r(x)} \leq c\|u\|_{X}
$$

That is, if $1<r(x)<p_{s}^{*}(x)$ for all $x \in \bar{\Omega}$ then the space $X$ is continuously embedded in $L^{r(x)}(\Omega)$. Moreover, this embedding is compact. In addition, when one considers functions $u \in X_{0}=W_{0}^{s, q(x), p(x, y)}(\Omega)$, it holds that

$$
|u|_{r(x)} \leq c\|u\| .
$$

Proposition 2.4. (see [8]) For the operator $\mathcal{L}_{p(x, y)}: X_{0} \rightarrow X_{0}^{*}$ defined as in the beginning of the paper, we have

$$
\left\langle\mathcal{L}_{p(x, y)} u, \varphi\right\rangle=\int_{\Omega \times \Omega} \frac{|u(x)-u(y)|^{p(x, y)-2}(u(x)-u(y))(\varphi(x)-\varphi(y))}{|x-y|^{N+s p(x, y)}} d x d y
$$

for all $\varphi \in X_{0}$. Moreover, under the conditions of Proposition 2.3, the following assertions hold true:

(i) $\mathcal{L}_{p(x, y)}$ is a bounded and strictly monotone operator.

(ii) $\mathcal{L}_{p(x, y)}$ is a mapping of type $\left(S_{+}\right)$, i.e., if $u_{n} \rightarrow u$ in $X_{0}$ and

$$
\limsup _{n \rightarrow \infty}\left\langle\mathcal{L}_{p(x, y)} u_{n}-\mathcal{L}_{p(x, y)} u, u_{n}-u\right\rangle=0
$$

then $u_{n} \rightarrow u$ in $X_{0}$ as $n \rightarrow \infty$.

(iii) $\mathcal{L}_{p(x, y)}$ is a homeomorphism.

\section{Main results}

In this section, we will state and prove our main results. We denote by $c_{i}$ general positive number whose value may change from line to line. 
Definition 3.1. We say that $\lambda \in \mathbb{R}$ is an eigenvalue of problem (1.1) if there exists $u \in X_{0} \backslash\{0\}$ such that

$$
\begin{aligned}
& \int_{\Omega \times \Omega} \frac{|u(x)-u(y)|^{p(x, y)-2}(u(x)-u(y))(\varphi(x)-\varphi(y))}{|x-y|^{N+s p(x, y)}} d x d y \\
& +\int_{\Omega}|u|^{q(x)-2} u \varphi d x-\lambda \int_{\Omega} V(x)|u|^{r(x)-2} u \varphi d x=0
\end{aligned}
$$

for all $\varphi \in X_{0}$. It is clear that if $\lambda$ is an eigenvalue of problem (1.1) then the corresponding eigenfunction $\varphi \in X_{0} \backslash\{0\}$ is a weak solution of problem (1.1).

Our first result concerns problem 1.1 in the sublinear case, we establish the existence of a continuous family of eigenvalues for problem $[1.1$ in a neighborhood of the origin.

Theorem 3.2. Let the functions $p, q: \bar{\Omega} \rightarrow \mathbb{R}$ be such that condition $(1.2)$ holds. Moreover, we assume that the functions $p: \bar{\Omega} \times \bar{\Omega} \rightarrow \mathbb{R}$, and $q, r, \sigma: \bar{\Omega} \rightarrow \mathbb{R}$ satisfy the following conditions:

$\left(\mathrm{H}_{1}\right) 1<r(x)<p^{-} \leq p^{+}<N / s<\sigma(x)$ for all $x \in \bar{\Omega}$;

$\left(\mathrm{H}_{2}\right) V \in L^{\sigma(x)}(\Omega)$ and there exists a measurable set $\Omega_{0} \subset \subset \Omega$ of positive measure such that $V(x)>0$ for all $x \in \Omega_{0}$.

Then there exists $\bar{\lambda}>0$ such that any $\lambda \in(0, \bar{\lambda})$ is an eigenvalue of problem (1.1).

Proof. For each $\lambda>0$, let us consider the functional $J_{\lambda}: X_{0} \rightarrow \mathbb{R}$ associated with problem (1.1) by the formula

$$
J_{\lambda}(u)=\Phi(u)-\lambda \Psi(u)
$$

where

$$
\begin{aligned}
\Phi(u) & =\int_{\Omega \times \Omega} \frac{|u(x)-u(y)|^{p(x, y)}}{p(x, y)|x-y|^{N+s p(x, y)}} d x d y+\int_{\Omega} \frac{1}{q(x)}|u|^{q(x)} d x, \\
\Psi(u) & =\int_{\Omega} \frac{V(x)}{r(x)}|u|^{r(x)} d x .
\end{aligned}
$$

From conditions $\left(\mathrm{H}_{1}\right)-\left(\mathrm{H}_{2}\right)$ and Proposition 2.1, for all $u \in X_{0}$, we get

$$
\begin{aligned}
|\Psi(u)| & \leq\left.\left.\frac{2}{r^{-}}|V|_{\sigma(x)}|| u\right|^{r(x)}\right|_{\sigma(x) /(\sigma(x)-1)} \\
& \leq \begin{cases}\frac{2}{r^{-}}|V|_{\sigma(x)}|u|_{\sigma(x) r(x) /(\sigma(x)-1)}^{r^{-}} & \text {if }|u|_{\sigma(x) r(x) /(\sigma(x)-1)} \leq 1, \\
\frac{2}{r^{-}}|V|_{\sigma(x)}|u|_{\sigma(x) r(x) /(\sigma(x)-1)}^{r^{+}} & \text {if }|u|_{\sigma(x) r(x) /(\sigma(x)-1)} \geq 1 .\end{cases}
\end{aligned}
$$

We also deduce from $\left(\mathrm{H}_{1}\right)$ that $\alpha(x)=\sigma(x) r(x) /(\sigma(x)-1)<p_{s}^{*}(x)$ and $\beta(x)=$ $\sigma(x) r(x) /(\sigma(x)-r(x))<p_{s}^{*}(x)$ for all $x \in \bar{\Omega}$ and by Proposition 2.3, the embeddings 
$X_{0} \hookrightarrow L^{\alpha(x)}(\Omega)$ and $X_{0} \hookrightarrow L^{\beta(x)}(\Omega)$ are continuous and compact. Thus, the functional $J_{\lambda}$ is well-defined on $X_{0}$. The proof of Theorem 3.2 is divided into the following four steps.

Step 1 . We prove that $J_{\lambda} \in C^{1}\left(X_{0}, \mathbb{R}\right)$ and its derivative is

$$
\begin{aligned}
J_{\lambda}^{\prime}(u)(\varphi)= & \int_{\Omega \times \Omega} \frac{|u(x)-u(y)|^{p(x, y)-2}(u(x)-u(y))(\varphi(x)-\varphi(y))}{|x-y|^{N+s p(x, y)}} d x d y \\
& +\int_{\Omega}|u|^{q(x)-2} u \varphi d x-\lambda \int_{\Omega} V(x)|u|^{r(x)-2} u \varphi d x
\end{aligned}
$$

for all $u, \varphi \in X_{0}$. This means that weak solutions of problem (1.1) can be found as the critical points of the functional $J_{\lambda}$ in the space $X_{0}$.

First of all, using the same method as in the proof of [8, Lemma 4.1], we can show that $\Phi \in C^{1}\left(X_{0}, \mathbb{R}\right)$ and

$$
\begin{aligned}
\Phi^{\prime}(u)(\varphi)= & \int_{\Omega \times \Omega} \frac{|u(x)-u(y)|^{p(x, y)-2}(u(x)-u(y))(\varphi(x)-\varphi(y))}{|x-y|^{N+s p(x, y)}} d x d y \\
& +\int_{\Omega}|u|^{q(x)-2} u \varphi d x
\end{aligned}
$$

for all $u, \varphi \in X_{0}$.

Now, we will prove that $\Psi \in C^{1}\left(X_{0}, \mathbb{R}\right)$, that is, for given $u \in X_{0}$ we show that for all $\varphi \in X_{0}$, it holds that

$$
\lim _{t \rightarrow 0} \frac{\Psi(u+t \varphi)-\Psi(u)}{t}=\Psi^{\prime}(u)(\varphi)
$$

and $\Psi^{\prime}: X_{0} \rightarrow X_{0}^{*}$ is continuous, where we denote by $X_{0}^{*}$ the dual space of $X_{0}$.

Indeed, by conditions $\left(\mathrm{H}_{1}\right)-\left(\mathrm{H}_{2}\right)$, for $|t|<1$ using Hölder's inequality (2.2) and Propositions 2.1 and 2.3 , it implies that

$$
\begin{aligned}
\int_{\Omega}|V(x)| u+\left.t \varphi\right|^{r(x)-2}(u+t \varphi) \varphi \mid d x & \leq \int_{\Omega}|V(x)||u+t \varphi|^{r(x)-1}|\varphi| d x \\
& \leq \int_{\Omega}|V(x)|(|u|+|\varphi|)^{r(x)-1}|\varphi| d x \\
& \leq 3|V(x)|_{\sigma(x)}|||u|+\left.\left.|\varphi|\right|^{r(x)-1}\right|_{r(x) /(r(x)-1)}|\varphi|_{\beta(x)} \\
& \leq 3|V|_{\sigma(x)}|| u|+| \varphi||_{r(x)}^{r^{\tau}-1}|\varphi|_{\beta(x)} \\
& <+\infty
\end{aligned}
$$

where $\tau=+$ if $\left.|| u|+| \varphi\right|_{r(x)}>1$ and $\tau=-$ if ||$u|+| \varphi \|_{r(x)} \leq 1$ since $X_{0} \hookrightarrow L^{\beta(x)}(\Omega)$, $X_{0} \hookrightarrow L^{r(x)}(\Omega)$ and $V \in L^{\sigma(x)}(\Omega)$. 
For all $\varphi \in X_{0}$, using Lebesgue's convergence theorem, we get

$$
\begin{aligned}
& \lim _{t \rightarrow 0} \frac{\Psi(u+t \varphi)-\Psi(u)}{t} \\
= & \left.\frac{d}{d t} \Psi(u+t \varphi)\right|_{t=0}=\left.\left(\frac{d}{d t} \int_{\Omega} \frac{V(x)}{r(x)}|u+t \varphi|^{r(x)} d x\right)\right|_{t=0} \\
= & \left.\int_{\Omega} \frac{d}{d t}\left(\int_{\Omega} \frac{V(x)}{r(x)}|u+t \varphi|^{r(x)} d x\right)\right|_{t=0} d x=\left.\int_{\Omega} V(x)|u+t \varphi|^{r(x)-2}(u+t \varphi) \varphi\right|_{t=0} d x \\
= & \int_{\Omega} V(x)|u|^{r(x)-2} u \varphi d x=\Psi^{\prime}(u)(\varphi) .
\end{aligned}
$$

Since the embedding $X_{0} \hookrightarrow L^{\beta(x)}(\Omega)$ is continuous, there exists $c_{1}>0$ such that $|\varphi|_{\beta(x)} \leq c_{1}\|\varphi\|$ for all $\varphi \in X_{0}$ and by conditions $\left(\mathrm{H}_{1}\right)-\left(\mathrm{H}_{2}\right)$,

$$
\begin{aligned}
\left|\Psi^{\prime}(u)(\varphi)\right| & =\left.\left.\left|\int_{\Omega} V(x)\right| u\right|^{r(x)-2} u \varphi d x\left|\leq \int_{\Omega}\right| V(x)|| u\right|^{r(x)-1}|\varphi| d x \\
& \leq 3|V|_{\sigma(x)}|u|_{r(x)}^{r^{\tau}-1}|\varphi|_{\beta(x)} \leq 3 c_{1}|V|_{\sigma(x)}|u|_{r(x)}^{r^{\tau}-1}\|\varphi\|
\end{aligned}
$$

for any $\varphi \in X_{0}$, where $\tau=+$ if $|u|_{r(x)}>1$ and $\tau=-$ if $|u|_{r(x)} \leq 1$. Combining this with the linearity of $\Psi^{\prime}$ we deduce that $\Psi^{\prime} \in X_{0}^{*}$. Note that the map $u \mapsto|u|^{r(x)-2} u$ from $L^{r(x)}(\Omega)$ into $L^{r(x) /(r(x)-1)}(\Omega)$ is continuous. For the Fréchet differentiability, we conclude that $\Psi$ is Fréchet differentiable and

$$
\Psi^{\prime}(u)(\varphi)=\int_{\Omega} V(x)|u|^{r(x)-2} u \varphi d x, \quad \forall u, \varphi \in X_{0}
$$

and thus Step 1 is completed.

Step 2. We prove that there exists $\bar{\lambda}>0$ such that for any $\lambda \in(0, \bar{\lambda})$, there exist constants $R, \rho>0$ such that $J_{\lambda}(u) \geq R$ for all $u \in X_{0}$ with $\|u\|=\rho$.

Indeed, since $\alpha(x)=\sigma(x) r(x) /(\sigma(x)-1)<p^{*}(x)$ for all $x \in \bar{\Omega}$, the embedding $X_{0} \hookrightarrow L^{\alpha(x)}(\Omega)$ is continuous, there exists $c_{2}>0$ such that

$$
|u|_{\alpha(x)} \leq c_{2}\|u\|, \quad \forall u \in X_{0}
$$

Hence, by relation (3.1), for any $u \in X_{0}$ with $\|u\|=\rho$ small enough,

$$
\begin{aligned}
J_{\lambda}(u) & =\int_{\Omega \times \Omega} \frac{|u(x)-u(y)|^{p(x, y)}}{p(x, y)|x-y|^{N+s p(x, y)}} d x d y+\int_{\Omega} \frac{1}{q(x)}|u|^{q(x)} d x-\lambda \int_{\Omega} \frac{V(x)}{r(x)}|u|^{r(x)} d x \\
& \geq \frac{1}{p^{+}}\|u\|^{p^{+}}-\lambda \frac{2 c_{2}^{r^{-}}}{r^{-}}|V|_{\sigma(x)}\|u\|^{r^{-}}=\frac{1}{p^{+}} \rho^{p^{+}}-\lambda \frac{2 c_{2}^{r^{-}}}{r^{-}}|V|_{\sigma(x)} \rho^{r^{-}} \\
& =\rho^{r^{-}}\left(\frac{1}{p^{+}} \rho^{p^{+}-r^{-}}-\lambda \frac{2 c_{2}^{r^{-}}}{r^{-}}|V|_{\sigma(x)}\right) .
\end{aligned}
$$


Putting

$$
\bar{\lambda}=\frac{\rho^{p^{+}-r^{-}}}{2 p^{+}} \cdot \frac{r^{-}}{2 c_{2}^{r^{-}}|V|_{\sigma(x)}}>0,
$$

then for any $\lambda \in(0, \bar{\lambda})$ and $u \in X_{0}$ with $\|u\|=\rho$, there exists $R=\rho^{p^{+}} /\left(2 p^{+}\right)$such that $J_{\lambda}(u) \geq R>0$.

Step 3. We prove that there exists $\varphi_{0} \in X_{0}$ such that $\varphi_{0} \geq 0, \varphi_{0} \neq 0$ and $J_{\lambda}\left(t \varphi_{0}\right)<0$ for all $t>0$ small enough.

Indeed, condition $\left(\mathrm{H}_{1}\right)$ implies that $r(x)<\min \left\{p^{-}, q^{-}\right\}=p^{-}$for all $x \in \bar{\Omega}_{0}$. In the sequel, we use the notation $r_{0}^{-}:=\inf _{x \in \bar{\Omega}_{0}} r(x)$. Let $\epsilon_{0}>0$ be such that $r_{0}^{-}+\epsilon_{0}<p^{-}$. We also have since $r \in C\left(\bar{\Omega}_{0}\right)$ that there exists an open subset $\Omega_{1} \subset \Omega_{0}$ such that

$$
\left|r(x)-r_{0}^{-}\right|<\epsilon_{0}, \quad \forall x \in \Omega_{1}
$$

and thus

$$
r(x) \leq r_{0}^{-}+\epsilon_{0}<p^{-}, \quad \forall x \in \Omega_{1} .
$$

Let $\varphi_{0} \in C_{0}^{\infty}\left(\Omega_{0}\right)$ such that $\bar{\Omega}_{1} \subset \operatorname{supp}\left(\varphi_{0}\right), \varphi_{0}(x)=1$ for all $x \in \bar{\Omega}_{1}$ and $0 \leq \varphi_{0} \leq 1$ in $\Omega_{0}$. Then, using the above information, for any $t \in(0,1)$ we have

$$
\begin{aligned}
J_{\lambda}\left(t \varphi_{0}\right)= & \int_{\Omega \times \Omega} \frac{\left|t \varphi_{0}(x)-t \varphi_{0}(y)\right|^{p(x, y)}}{p(x, y)|x-y|^{N+s p(x, y)}} d x d y+\int_{\Omega} \frac{1}{q(x)}\left|t \varphi_{0}\right|^{q(x)} d x-\lambda \int_{\Omega} \frac{V(x)}{r(x)}\left|t \varphi_{0}\right|^{r(x)} d x \\
\leq & \frac{t^{p^{-}}}{p^{-}} \int_{\Omega_{0} \times \Omega_{0}} \frac{\left|\varphi_{0}(x)-\varphi_{0}(y)\right|^{p(x, y)}}{|x-y|^{N+s p(x, y)}} d x d y+\frac{t^{q^{-}}}{q^{-}} \int_{\Omega_{0}}\left|\varphi_{0}\right|^{q(x)} d x \\
& -\lambda \int_{\Omega_{0}} \frac{V(x)}{r(x)} t^{r(x)}\left|\varphi_{0}\right|^{r(x)} d x \\
\leq & \frac{t^{p^{-}}}{p^{-}}\left(\int_{\Omega_{0} \times \Omega_{0}} \frac{\left|\varphi_{0}(x)-\varphi_{0}(y)\right|^{p(x, y)}}{|x-y|^{N+s p(x, y)}} d x d y+\int_{\Omega_{0}}\left|\varphi_{0}\right|^{q(x)} d x\right) \\
& -\frac{\lambda t^{r_{0}^{-}+\epsilon_{0}}}{r_{0}^{+}} \int_{\Omega_{1}} V(x)\left|\varphi_{0}\right|^{r(x)} d x .
\end{aligned}
$$

Therefore, $J_{\lambda}\left(t \varphi_{0}\right)<0$ for $0<t<\delta^{1 /\left(p^{-}-r_{0}^{-}-\epsilon_{0}\right)}$ with

$$
0<\delta<\min \left\{1, \frac{\lambda p^{-}}{r_{0}^{+}} \cdot \frac{\int_{\Omega_{1}} V(x)\left|\varphi_{0}\right|^{r(x)} d x}{\int_{\Omega \times \Omega} \frac{\left|\varphi_{0}(x)-\varphi_{0}(y)\right|^{p(x, y)}}{|x-y|^{N+s(x, y)}} d x d y+\int_{\Omega}\left|\varphi_{0}\right|^{q(x)} d x}\right\}
$$

The above fraction is meaningful if we can show that

$$
\int_{\Omega \times \Omega} \frac{\left|\varphi_{0}(x)-\varphi_{0}(y)\right|^{p(x, y)}}{|x-y|^{N+s p(x, y)}} d x d y+\int_{\Omega}\left|\varphi_{0}\right|^{q(x)} d x>0 .
$$

Indeed, it is clear that

$$
\int_{\Omega_{1}}\left|\varphi_{0}\right|^{r(x)} d x \leq \int_{\Omega}\left|\varphi_{0}\right|^{r(x)} d x \leq \int_{\Omega}\left|\varphi_{0}\right|^{r^{-}} d x .
$$


On the other hand, the space $X_{0}$ is continuously embedded in $L^{r^{-}}(\Omega)$ and thus, there exists $c_{3}>0$ such that $\left|\varphi_{0}\right|_{r^{-}} \leq c_{3}\left\|\varphi_{0}\right\|$, which implies that $\left\|\varphi_{0}\right\|>0$. Combining this with 2.3 or (2.4) the claim follows at once.

By Step 2, we have

$$
\inf _{u \in \partial B_{\rho}(0)} J_{\lambda}(u)>0 .
$$

We also deduce from Step 2 that, the functional $J_{\lambda}$ is bounded from below on $B_{\rho}(0)$. Moreover, by Step 3, there exists $\varphi \in X$ such that $J_{\lambda}(t \varphi)<0$ for all $t>0$ small enough. It follows from Step 2 that

$$
J_{\lambda}(u) \geq \frac{1}{p^{+}}\|u\|^{p^{+}}-\lambda \frac{2 c_{2}^{r^{-}}}{r^{-}}|V|_{\sigma(x)}\|u\|^{r^{-}},
$$

which yields

$$
-\infty<\underline{c}_{\lambda}=\inf _{u \in \bar{B}_{\rho}(0)} J_{\lambda}(u)<0 .
$$

Let us choose $\epsilon>0$ such that $0<\epsilon<\inf _{u \in \partial B_{\rho}(0)} J_{\lambda}(u)-\inf _{u \in \bar{B}_{\rho}(0)} J_{\lambda}(u)$. Applying the Ekeland variational principle 17 to the functional $J_{\lambda}: \bar{B}_{\rho}(0) \rightarrow \mathbb{R}$, it follows that there exists $u_{\epsilon} \in \bar{B}_{\rho}(0)$ such that

$$
J_{\lambda}\left(u_{\epsilon}\right)<\inf _{u \in \bar{B}_{\rho}(0)} J_{\lambda}(u)+\epsilon, \quad J_{\lambda}\left(u_{\epsilon}\right)<J_{\lambda}(u)+\epsilon\left\|u-u_{\epsilon}\right\|, \quad u \neq u_{\epsilon}
$$

then we infer that $J_{\lambda}\left(u_{\epsilon}\right)<\inf _{u \in \partial B_{\rho}(0)} J_{\lambda}(u)$, and thus $u_{\epsilon} \in B_{\rho}(0)$.

Let us consider the functional $I_{\lambda}: \bar{B}_{\rho}(0) \rightarrow \mathbb{R}$ by $I_{\lambda}(u)=J_{\lambda}(u)+\epsilon\left\|u-u_{\epsilon}\right\|$. Then $u_{\epsilon}$ is a minimum point of $I_{\lambda}$ and thus

$$
\frac{I_{\lambda}\left(u_{\epsilon}+\tau \varphi\right)-I_{\lambda}\left(u_{\epsilon}\right)}{t} \geq 0
$$

for all $\tau>0$ small enough and all $v \in B_{\rho}(0)$. The above information shows that

$$
\frac{J_{\lambda}\left(u_{\epsilon}+\tau \varphi\right)-J_{\lambda}\left(u_{\epsilon}\right)}{\tau}+\epsilon\|\varphi\| \geq 0 .
$$

Letting $\tau \rightarrow 0^{+}$, we deduce that

$$
J_{\lambda}^{\prime}\left(u_{\epsilon}\right)(\varphi)+\epsilon\|\varphi\| \geq 0
$$

and we infer that $\left\|J_{\lambda}^{\prime}\left(u_{\epsilon}\right)\right\|_{X_{0}^{*}} \leq \epsilon$. Therefore, there exists a sequence $\left\{u_{n}\right\} \subset B_{\rho}(0)$ such that

$$
J_{\lambda}\left(u_{n}\right) \rightarrow \underline{c}=\inf _{u \in \bar{B}_{\rho}(0)} J_{\lambda}(u)<0 \quad \text { and } \quad J_{\lambda}^{\prime}\left(u_{n}\right) \rightarrow 0 \quad \text { in } X_{0}^{*} \text { as } n \rightarrow \infty .
$$

Is is clear that the sequence $\left\{u_{n}\right\}$ is bounded in $X_{0}$. Now, since $X_{0}$ is a reflexive Banach space, there exists $u \in X_{0}$ such that passing to a subsequence, still denoted by $\left\{u_{n}\right\}$, it converges weakly to $u$ in $X_{0}$. 
Step 4. We prove that $\left\{u_{n}\right\}$ which is given by (3.2) converges strongly to $u$ in $X_{0}$, i.e., $\lim _{n \rightarrow \infty}\left\|u_{n}-u\right\|=0$.

By conditions $\left(\mathrm{H}_{1}\right)-\left(\mathrm{H}_{2}\right)$, using Hölder's inequality 2.2$)$ and Propositions 2.1 and 2.3 we deduce that

$$
\begin{aligned}
\left.\left|\int_{\Omega}\right| u_{n}\right|^{q(x)-2} u_{n}\left(u_{n}-u\right) d x \mid & \leq\left.\left. 2|| u_{n}\right|^{q(x)-2} u_{n}\right|_{q(x) /(q(x)-1)}\left|u_{n}-u\right|_{q(x)} \\
& \leq 2\left|u_{n}\right|_{q(x)}^{q^{+}-1}\left|u_{n}-u\right|_{q(x)} \\
& \rightarrow 0 \text { as } n \rightarrow \infty
\end{aligned}
$$

and

$$
\begin{aligned}
\left.\left|\int_{\Omega} V(x)\right| u_{n}\right|^{r(x)-2} u_{n}\left(u_{n}-u\right) d x \mid & \leq\left.\left. 3|V|_{\sigma(x)}|| u_{n}\right|^{r(x)-2} u_{n}\right|_{r(x) /(r(x)-1)}\left|u_{n}-u\right|_{\beta(x)} \\
& \leq 3|V|_{\sigma(x)}\left(1+\left|u_{n}\right|_{r(x)}^{r^{+}-1}\right)\left|u_{n}-u\right|_{\beta(x)} \\
& \rightarrow 0 \quad \text { as } n \rightarrow \infty,
\end{aligned}
$$

where $\beta(x)=\sigma(x) r(x) /(\sigma(x)-r(x))$. Moreover, by (3.2) we have $\lim _{n \rightarrow \infty} J_{\lambda}^{\prime}\left(u_{n}\right)\left(u_{n}-u\right)=$ 0, i.e.,

$$
\begin{aligned}
& \int_{\Omega \times \Omega} \frac{\left|u_{n}(x)-u_{n}(y)\right|^{p(x, y)-2}\left(u_{n}(x)-u_{n}(y)\right)\left(\left(u_{n}(x)-u(x)\right)-\left(u_{n}(y)-u(y)\right)\right)}{|x-y|^{N+s p(x, y)}} d x d y \\
+ & \int_{\Omega}\left|u_{n}\right|^{q(x)-2} u_{n}\left(u_{n}-u\right) d x-\lambda \int_{\Omega} V(x)\left|u_{n}\right|^{r(x)-2} u_{n}\left(u_{n}-u\right) d x \rightarrow 0 \quad \text { as } n \rightarrow \infty,
\end{aligned}
$$

which yields

$$
\int_{\Omega \times \Omega} \frac{\left|u_{n}(x)-u_{n}(y)\right|^{p(x, y)-2}\left(u_{n}(x)-u_{n}(y)\right)\left(\left(u_{n}(x)-u(x)\right)-\left(u_{n}(y)-u(y)\right)\right)}{|x-y|^{N+s p(x, y)}} d x d y \rightarrow 0
$$

or

$$
\lim _{n \rightarrow \infty} \mathcal{L}_{p(x, y)}\left(u_{n}\right)\left(u_{n}-u\right)=0
$$

Since $\left\{u_{n}\right\}$ converges weakly to $u$ in $X_{0}$, we also have that $\lim _{n \rightarrow \infty} \mathcal{L}_{p(x, y)}(u)\left(u_{n}-u\right)=0$. Combining this with 3.3 we get

$$
\lim _{m \rightarrow \infty}\left(\mathcal{L}_{p(x, y)}\left(u_{n}\right)-\mathcal{L}_{p(x, y)}(u)\right)\left(u_{n}-u\right)=0 .
$$

Relation (3.4) and Proposition 2.4 show actually the sequence $\left\{u_{n}\right\}$ converges strongly to $u$ in $X_{0}$. Thus, in view of $(3.2)$, we obtain $J_{\lambda}(u)=\underline{c}_{\lambda}<0$ and $J_{\lambda}^{\prime}(u)=0$. This means that $u$ is a non-trivial weak solution of $(1.1)$, i.e., any $\lambda \in(0, \bar{\lambda})$ is an eigenvalue of problem (1.1). Theorem 3.2 is completely proved. 
Now we will prove the second main result for problem (1.1) regarding the superlinear case. We also consider $V$ as an indefinite weight in the sense that it is allowed to change the sign in the domain $\Omega$. Let us define the subsets of $X$ as follows:

$$
\begin{aligned}
& X_{0}^{+}:=\left\{u \in X_{0}: \int_{\Omega} V(x)|u|^{r(x)} d x>0\right\}, \\
& X_{0}^{-}:=\left\{u \in X_{0}: \int_{\Omega} V(x)|u|^{r(x)} d x<0\right\},
\end{aligned}
$$

and

$$
\begin{aligned}
& \lambda^{*}=\inf _{u \in X_{0}^{+}} \frac{\Phi(u)}{\Psi(u)}, \quad \lambda_{*}=\inf _{u \in X_{0}^{+}} \frac{\int_{\Omega \times \Omega} \frac{|u(x)-u(y)|^{p(x, y)}}{|x-y|^{N+s p(x, y)}} d x d y+\int_{\Omega}|u|^{q(x)} d x}{\int_{\Omega} V(x)|u|^{r(x)} d x}, \\
& \mu^{*}=\sup _{u \in X_{0}^{-}} \frac{\Phi(u)}{\Psi(u)}, \quad \mu_{*}=\sup _{u \in X_{0}^{-}} \frac{\int_{\Omega \times \Omega} \frac{|u(x)-u(y)|^{p(x, y)}}{|x-y|^{N+s(x, y)}} d x d y+\int_{\Omega}|u|^{q(x)} d x}{\int_{\Omega} V(x)|u|^{r(x)} d x} .
\end{aligned}
$$

Theorem 3.3. Let the functions $p, q: \bar{\Omega} \rightarrow \mathbb{R}$ be such that condition 1.2 holds. Moreover, we assume that the functions $p: \bar{\Omega} \times \bar{\Omega} \rightarrow \mathbb{R}$, and $q, r, \sigma: \bar{\Omega} \rightarrow \mathbb{R}$ satisfy the following conditions:

$\left(\mathrm{H}_{3}\right) 1<\min \left\{p^{-}, q^{-}\right\} \leq \max \left\{p^{+}, q^{+}\right\}<r(x)<p_{s}^{*}(x)$ for all $x \in \bar{\Omega}$, and $r^{+}-1 / 2<r^{-}$;

$\left(\mathrm{H}_{4}\right) V \in L^{\sigma(x)}(\Omega)$ is a changing sign function such that $\sigma \in C_{+}(\bar{\Omega})$ and

$$
\sigma(x)>\max \left\{1, \frac{N p(x, x)}{N p(x, x)+s p(x, x) r(x)-N r(x)}\right\}, \quad \forall x \in \bar{\Omega} .
$$

Then we have

(i) $\lambda^{*}$ and $\mu^{*}$ are the positive and negative eigenvalue of problem (1.1) respectively, satisfying $\mu^{*} \leq \mu_{*}<0<\lambda_{*} \leq \lambda^{*}$;

(ii) $\lambda \in\left(-\infty, \mu^{*}\right) \cup\left(\lambda^{*},+\infty\right)$ is an eigenvalue of problem (1.1) while any $\lambda \in\left(\mu_{*}, \lambda_{*}\right)$ is not an eigenvalue.

Proof. Note that if $\lambda$ is an eigenvalue of problem 1.1 with weight $V$ then $-\lambda$ is an eigenvalue of problem (1.1) with weight $-V$. Hence, it is sufficient to prove Theorem 3.3 only for $\lambda>0$ and we will consider problem (1.1) only in the set $X_{0}^{+}$defined as before. For this case, the proof of Theorem 3.3 is divided into the following four steps.

Step 1. We prove that $\lambda_{*}>0$.

By relation (3.5), it follows that

$$
\frac{r^{-}}{\max \left\{p^{+}, q^{+}\right\}} \lambda_{*} \leq \lambda^{*} \leq \frac{r^{+}}{\min \left\{p^{-}, q^{-}\right\}} \lambda_{*}=\frac{r^{+}}{p^{-}} \lambda_{*},
$$


and $0 \leq \lambda_{*} \leq \lambda^{*}$ since $\min \left\{p^{-}, q^{-}\right\}=p^{-}$and $\max \left\{p^{+}, q^{+}\right\}<r^{-}$, see conditions (1.2) and $\left(\mathrm{H}_{3}\right)$.

We will prove that

$$
\begin{aligned}
\lim _{\|u\| \rightarrow 0, u \in X_{0}^{+}} \frac{\Phi(u)}{\Psi(u)} & =+\infty, \\
\lim _{\|u\| \rightarrow+\infty, u \in X_{0}^{+}} \frac{\Phi(u)}{\Psi(u)} & =+\infty .
\end{aligned}
$$

Indeed, first of all, we will prove (3.7) holds. Using Hölder's inequality 2.1) and Proposition 2.1, for all $u \in X_{0}$ we have

$$
|\Psi(u)| \leq\left.\left.\frac{2}{r^{-}}|V|_{\sigma(x)}|| u\right|^{r(x)}\right|_{\sigma(x) /(\sigma(x)-1)} \leq \frac{2}{r^{-}}|V|_{\sigma(x)}|u|_{\alpha(x)}^{r^{\tau}},
$$

where $\tau=-$ if $|u|_{\alpha(x)} \leq 1$ and $\tau=+$ if $|u|_{\alpha(x)} \geq 1$ and $\alpha(x)=\sigma(x) r(x) /(\sigma(x)-1)$. By condition $\left(\mathrm{H}_{4}\right)$, we have $1<\alpha(x)<p_{s}^{*}(x)$ for all $x \in \bar{\Omega}$, that is, $X_{0}$ is continuously embedded in $L^{\alpha(x)}(\Omega)$, so there exists $c_{4}>0$ such that

$$
|\Psi(u)| \leq \frac{2 c_{4}}{r^{-}}|V|_{\sigma(x)}\|u\|^{r^{\tau}} .
$$

For all $u \in X_{0}^{+}$with $\|u\| \leq 1$ by relation $(3.9)$, we infer that

$$
\begin{aligned}
\frac{\Phi(u)}{\Psi(u)} & =\frac{\int_{\Omega \times \Omega} \frac{|u(x)-u(y)|^{p(x, y)}}{p(x, y)|x-y|^{N+s p(x, y)}} d x d y+\int_{\Omega} \frac{1}{q(x)}|u|^{q(x)} d x}{\int_{\Omega} \frac{V(x)}{r(x)}|u|^{r(x)} d x} \\
& \geq \frac{\int_{\Omega \times \Omega} \frac{|u(x)-u(y)|^{p(x, y)}}{p(x, y)|x-y|^{N+s p(x, y)}} d x d y}{\int_{\Omega} \frac{V(x)}{r(x)}|u|^{r(x)} d x} \\
& \geq \frac{r^{-}}{2 c_{4} p^{+}|V|_{\sigma(x)}}\|u\|^{p^{+}-r^{-}}
\end{aligned}
$$

which helps us to get relation (3.7) because of $\left(\mathrm{H}_{3}\right)$, that is, $p^{+}<r^{-}$.

On the other hand, since $r^{+}-1 / 2<r^{-}$, there exists a constant $\theta>0$ such that $r^{+}-1 / 2<\theta<r^{-}$, which gives us $r^{+}-1<r^{-}-1 / 2<\theta$ and

$$
1+\theta-r^{+}>0, \quad 2\left(r^{-}-\theta\right) \leq 2\left(r^{+}-\theta\right)<1 .
$$

Let us take $\gamma(x)$ any measurable function satisfying

$$
\max \left\{\frac{\sigma(x)}{1+\theta \sigma(x)}, \frac{p_{s}^{*}(x)}{p_{s}^{*}(x)+\theta-r(x)}\right\}<\gamma(x)<\min \left\{\frac{p_{s}^{*}(x)}{p_{s}^{*}(x)+\theta \sigma(x)}, \frac{1}{1+\theta-r(x)}\right\}
$$

for all $x \in \bar{\Omega}$ and

$$
\theta\left(\frac{\gamma^{+}}{\gamma^{-}}+1\right)<r^{-} .
$$


From relations 3.11-3.13), it implies that $\gamma \in L^{\infty}(\Omega)$ and $1<\gamma(x)<\sigma(x)$ for any $x \in \bar{\Omega}$. Moreover, we have

$$
1<\frac{\theta \gamma(x) \sigma(x)}{\sigma(x)-\gamma(x)}<p_{s}^{*}(x), \quad 1<\frac{(r(x)-\theta) \gamma(x)}{\gamma(x)-1}<p_{s}^{*}(x), \quad \forall x \in \bar{\Omega}
$$

so there exists $c_{5}>0$ such that

$$
|u|_{\theta \gamma(x) \sigma(x) /(\sigma(x)-\gamma(x))} \leq c_{5}\|u\|, \quad|u|_{(r(x)-\theta) \gamma(x) /(\gamma(x)-1)} \leq c_{5}\|u\|, \quad \forall u \in X_{0} .
$$

For all $u \in X_{0}^{+}$, by Hölder's inequality 2.1 and 3.12 we have

$$
|\Psi(u)| \leq\left.\left.\int_{\Omega}|V| u\right|^{\theta}|| u\right|^{r(x)-\theta} d x \leq\left.\left.\left.\left. 2|V| u\right|^{\theta}\right|_{\gamma(x)}|| u\right|^{r(x)-\theta}\right|_{\gamma(x) /(\gamma(x)-1)}
$$

and

$$
\left.\left.|V| u\right|^{\theta}\right|_{\gamma(x)} \leq\left(\int_{\Omega}|V|^{\gamma(x)}|u|^{\theta \gamma(x)} d x\right)^{1 / \gamma^{-}} \leq\left.\left.\left.\left. 2|| V\right|^{\gamma(x)}\right|_{\sigma(x) / \gamma(x)} ^{1 / \gamma^{-}}|| u\right|^{\theta \gamma(x)}\right|_{\sigma(x) /(\sigma(x)-\gamma(x))} ^{1 / \gamma^{-}}
$$

for all $u \in X_{0}^{+}$with $\left.\left.|V| u\right|^{\theta}\right|_{\gamma(x)}>1$. Thus, by (3.14, we infer that

$$
\begin{aligned}
\left.\left.|V| u\right|^{\theta}\right|_{\gamma(x)} & \leq 1+\left.\left.\left.\left.2|| V\right|^{\gamma(x)}\right|_{\sigma(x) / \gamma(x)} ^{1 / \gamma^{-}}|| u\right|^{\theta \gamma(x)}\right|_{\sigma(x) /(\sigma(x)-\gamma(x))} ^{1 / \gamma^{-}} \\
& \leq 1+2\left(1+|V|_{\gamma(x)}^{\gamma^{+} / \gamma^{-}}\right)\left(1+|u|_{\theta \gamma(x) \sigma(x) /(\sigma(x)-\gamma(x))}^{\theta \gamma^{+} / \gamma^{-}}\right) \\
& \leq c_{6}\left(1+\|u\|^{\theta \gamma^{+} / \gamma^{-}}\right)
\end{aligned}
$$

for any $u \in X_{0}^{+}$. Similarly,

$$
\left.\left.|| u\right|^{r(x)-\theta}\right|_{\gamma(x) /(\gamma(x)-1)} \leq 1+|u|_{\gamma(x)(r(x)-\theta) /(\gamma(x)-1)}^{r^{+}-\theta} \leq 1+c_{7}\|u\|^{r^{+}-\theta}, \quad \forall u \in X_{0}^{+} .
$$

Combining (3.14) with the above information, we deduce that

$$
\begin{aligned}
|\Psi(u)| & \leq c_{6}\left(1+\|u\|^{\theta \gamma^{+} / \gamma^{-}}\right)\left(1+c_{7}\|u\|^{r^{+}-\theta}\right) \\
& =c_{6}+c_{6} c_{7}\|u\|^{r^{+}-\theta}+c_{6}\|u\|^{\theta \gamma^{+} / \gamma^{-}}+c_{6} c_{7}\|u\|^{\theta \gamma^{+} / \gamma^{-}}\|u\|^{r^{+}-\theta} \\
& \leq c_{8}\left(1+\|u\|^{2 \theta \gamma^{+} / \gamma^{-}}+\|u\|^{2\left(r^{+}-\theta\right)}\right)
\end{aligned}
$$

for all $u \in X_{0}^{+}$with $\|u\|>1$. 
Hence,

$$
\begin{aligned}
\frac{\Phi(u)}{\Psi(u)} & =\frac{\int_{\Omega \times \Omega} \frac{|u(x)-u(y)|^{p(x, y)}}{p(x, y)|x-y|^{N+s p(x, y)}} d x d y+\int_{\Omega} \frac{1}{q(x)}|u|^{q(x)} d x}{\int_{\Omega} \frac{V(x)}{r(x)}|u|^{r(x)} d x} \\
& \geq \frac{\int_{\Omega \times \Omega} \frac{|u(x)-u(y)|^{p(x, y)}}{p(x, y)|x-y|^{N+s p(x, y)}} d x d y}{\int_{\Omega} \frac{V(x)}{r(x)}|u|^{r(x)} d x} \\
& \geq \frac{\frac{1}{p^{+}}\|u\|^{p^{-}}}{c_{8}\left(1+\|u\|^{2 \theta \gamma^{+} / \gamma^{-}}+\|u\|^{2\left(r^{+}-\theta\right)}\right)} \\
& \rightarrow+\infty
\end{aligned}
$$

as $\|u\| \rightarrow+\infty$ since $p^{-}>1>2\left(r^{+}-\theta\right) \geq 2\left(r^{-}-\theta\right) \geq 2 \theta \gamma^{+} / \gamma^{-}>2 \theta$. Thus relation (3.8) holds.

Now, we are in the position to prove that $\lambda_{*}>0$. Assume by contradiction that $\lambda_{*}=0$, from (3.6) we get $\lambda^{*}=0$. Then, there exists a sequence $\left\{u_{n}\right\} \subset X_{0}^{+} \backslash\{0\}$ such that

$$
\lim _{n \rightarrow \infty} \frac{\Phi\left(u_{n}\right)}{\Psi\left(u_{n}\right)}=0
$$

We also obtain from 3.10 that

$$
\begin{aligned}
\frac{\Phi\left(u_{n}\right)}{\Psi\left(u_{n}\right)} & \geq \frac{\int_{\Omega \times \Omega} \frac{\left|u_{n}(x)-u_{n}(y)\right|^{p(x, y)}}{p(x, y)|x-y|^{N+s p(x, y)}} d x d y}{\int_{\Omega} \frac{V(x)}{r(x)}\left|u_{n}\right|^{r(x)} d x} \\
& \geq \begin{cases}\frac{r^{-}}{2 c_{4} p^{+}|V|_{\sigma(x)}}\left\|u_{n}\right\|^{p^{-}-r^{+}} & \text {if }\left\|u_{n}\right\| \geq 1, \\
\frac{q^{-}}{2 c_{4} p^{+}|V|_{\sigma(x)}}\left\|u_{n}\right\|^{p^{+}-r^{-}} & \text {if }\left\|u_{n}\right\|<1 .\end{cases}
\end{aligned}
$$

By $\left(\mathrm{H}_{3}\right)$, we get $p^{-}-r^{+}<0$ and $p^{+}-r^{-}<0$, so (3.17) implies that $\left\|u_{n}\right\| \rightarrow+\infty$ as $n \rightarrow \infty$. Using again (3.17), we get

$$
\lim _{n \rightarrow \infty} \frac{\Phi\left(u_{n}\right)}{\Psi\left(u_{n}\right)}=+\infty,
$$

which contradicts 3.16 and thus, we have $\lambda_{*}>0$. The proof of Step 1 is completed.

Step 2. We prove that $\lambda^{*}$ is an eigenvalue of problem (1.1).

Indeed, let $\left\{u_{n}\right\} \subset X_{0}^{+} \backslash\{0\}$ be a minimizing sequence for the number $\lambda^{*}$, that is,

$$
\lim _{n \rightarrow \infty} \frac{\Phi\left(u_{n}\right)}{\Psi\left(u_{n}\right)}=\lambda^{*}>0 .
$$

By (3.18), we infer that $\left\{u_{n}\right\}$ is a bounded sequence in $X_{0}$. Since $X_{0}$ is reflexive, there exists $u^{*} \in X_{0}$ and a subsequence of $\left\{u_{n}\right\}$, still denoted by $\left\{u_{n}\right\}$ such that $\left\{u_{n}\right\}$ converges weakly to $u^{*}$ in $X_{0}$ as $n \rightarrow \infty$. Since $\Phi$ is weakly lower semi-continuous, we get

$$
\lim _{n \rightarrow \infty} \Phi\left(u_{n}\right) \geq \Phi\left(u^{*}\right) .
$$


On the other hand, since the embedding $X_{0} \hookrightarrow L^{\alpha(x)}(\Omega)$ is compact, the sequence $\left\{u_{n}\right\}$ converges strongly to $u^{*}$ in $L^{\alpha(x)}(\Omega)$, where $\alpha(x)=r(x) \sigma(x) /(\sigma(x)-1)$. It is noticed that $\left|u_{n}\right|_{\alpha(x)} \rightarrow\left|u^{*}\right|_{\alpha(x)},\left.\left.\left.\left.\quad|| u_{n}\right|^{r(x)}\right|_{\sigma(x) /(\sigma(x)-1)} \rightarrow|| u^{*}\right|^{r(x)}\right|_{\sigma(x) /(\sigma(x)-1)}$, the sequence $\left.\left.|| u_{n}\right|^{r(x)}\right|_{\sigma(x) /(\sigma(x)-1)}$ is bounded and $\left\{\left|u_{n}\right|^{r(x)}\right\}$ converges weakly to $\left|u^{*}\right|^{r(x)}$ in $L^{\sigma(x) /(\sigma(x)-1)}(\Omega)$, so we deduce that $\left|u_{n}\right|^{r(x)} \rightarrow\left|u^{*}\right|^{r(x)}$ in $L^{\sigma(x) /(\sigma(x)-1)}(\Omega)$. From the above information, it implies that

$$
\begin{aligned}
\left|\Psi\left(u_{n}\right)-\Psi\left(u^{*}\right)\right| & \leq \int_{\Omega} \frac{|V(x)|}{r(x)}\left(\left|u_{n}\right|^{r(x)}-\left|u^{*}\right|^{r(x)}\right) d x \\
& \leq\left.\frac{2}{r^{-}}|V|_{\sigma(x)}|| u_{n}\right|^{r(x)}-\left.\left|u^{*}\right|^{r(x)}\right|_{\sigma(x) /(\sigma(x)-1)} \\
& \rightarrow 0
\end{aligned}
$$

as $n \rightarrow \infty$, that is,

$$
\lim _{n \rightarrow \infty} \Psi\left(u_{n}\right)=\Psi\left(u^{*}\right)=\int_{\Omega} \frac{V(x)}{r(x)}\left|u^{*}\right|^{r(x)} d x \geq 0
$$

In view of (3.19) and (3.20) we obtain $\Phi\left(u^{*}\right) / \Psi\left(u^{*}\right)=\lambda^{*}$ if $\Psi\left(u^{*}\right)>0$, i.e., $u^{*} \in$ $X_{0}^{+} \backslash\{0\}$. We need to show that $\Psi\left(u^{*}\right)>0$. Assume by contradiction that $\Psi\left(u^{*}\right)=0$, that is, $\left\{\Psi\left(u_{n}\right)\right\}$ converges to 0 or

$$
\lim _{n \rightarrow \infty} \Psi\left(u_{n}\right)=0
$$

Now, taking $\epsilon \in\left(0, \lambda^{*}\right)$ be fixed, by (3.18), for $n$ large enough,

$$
\left|\frac{\Phi\left(u_{n}\right)}{\Psi\left(u_{n}\right)}-\lambda^{*}\right|<\epsilon
$$

or

$$
\left(\lambda^{*}-\epsilon\right) \Psi\left(u_{n}\right)<\Phi\left(u_{n}\right)<\left(\lambda^{*}+\epsilon\right) \Psi\left(u_{n}\right),
$$

which follows from (3.21) that $\lim _{n \rightarrow \infty} \Phi\left(u_{n}\right)=0$. This means that $u_{n} \rightarrow 0$ in $X_{0}$, that is, $\left\|u_{n}\right\| \rightarrow 0$ as $n \rightarrow \infty$ and thus,

$$
\lim _{n \rightarrow \infty} \frac{\Phi\left(u_{n}\right)}{\Psi\left(u_{n}\right)}=+\infty
$$

which is a contradiction. Therefore, $\Psi\left(u^{*}\right)>0$ and $u^{*} \in X_{0}^{+} \backslash\{0\}$ is an eigenfunction and $\lambda^{*}$ is an eigenvalue of problem (1.1).

Step 3. We prove that any $\lambda \in\left(\lambda^{*},+\infty\right)$ is an eigenvalue of problem (1.1).

Let $\lambda \in\left(\lambda^{*},+\infty\right)$ be arbitrary but fixed. We know that $\lambda$ is an eigenvalue of problem (1.1) if and only if there exists $u_{\lambda} \in X_{0}^{+} \backslash\{0\}$ a critical point of $J_{\lambda}$. 
From 3.15, it implies that

$$
\begin{aligned}
J_{\lambda}(u) & =\int_{\Omega \times \Omega} \frac{|u(x)-u(y)|^{p(x, y)}}{p(x, y)|x-y|^{N+s p(x, y)}} d x d y+\int_{\Omega} \frac{1}{q(x)}|u|^{q(x)} d x-\lambda \int_{\Omega} \frac{V(x)}{r(x)}|u|^{r(x)} d x \\
& \geq \frac{1}{p^{+}}\|u\|^{p^{-}}-\lambda c_{8}\left(1+\|u\|^{2 \theta \gamma^{+} / \gamma^{-}}+\|u\|^{2\left(r^{+}-\theta\right)}\right) \\
& \rightarrow+\infty
\end{aligned}
$$

as $\|u\| \rightarrow+\infty$ since $p^{-}>1>2\left(r^{+}-\theta\right) \geq 2\left(r^{-}-\theta\right) \geq 2 \theta \gamma^{+} / \gamma^{-}>2 \theta$. This follows that $J_{\lambda}$ is coercive in $X_{0}^{+}$.

By the of proof in Step 2, the functional $\Psi$ is weakly-strongly continuous in $X_{0}^{+}$. We also know that $\Phi$ is weakly lower semi-continuous, so by Weierstrass theorem, there exists $u_{\lambda} \in X_{0}^{+}$a global minimum point of $J_{\lambda}$. We need to prove that $u_{\lambda}$ is non-trivial critical point of $J_{\lambda}$. Indeed, since $\lambda^{*}=\inf _{u \in X_{0}^{+}} \Phi(u) / \Psi(u)$ and $\lambda>\lambda^{*}$, it follows that there exists $\bar{u}_{\lambda} \in X_{0}^{+}$such that $\Phi\left(\bar{u}_{\lambda}\right) / \Psi\left(\bar{u}_{\lambda}\right)<\lambda$, that is,

$$
J_{\lambda}\left(\bar{u}_{\lambda}\right)=\Phi\left(\bar{u}_{\lambda}\right)-\lambda \Psi\left(\bar{u}_{\lambda}\right)<0 .
$$

This means that inf ${ }_{u \in X_{0}^{+}} J_{\lambda}(u)<0$ and thus, $u_{\lambda}$ is a non-trivial critical point of $J_{\lambda}$ or $\lambda$ is an eigenvalue of problem (1.1). The proof of Step 3 is completed.

Step 4 . We prove that any $\lambda \in\left(0, \lambda_{*}\right)$ is not an eigenvalue of problem (1.1).

Indeed, assume by contradiction that there exists $\lambda \in\left(0, \lambda^{*}\right)$ is an eigenvalue of problem 1.1, that is, there exists $u_{\lambda} \in X_{0}^{+}$such that

$$
\Phi^{\prime}\left(u_{\lambda}\right)(v)=\lambda \Psi^{\prime}\left(u_{\lambda}\right)(v), \quad \forall v \in X_{0}^{+}
$$

Thus, for $v=u_{\lambda} \in X_{0}^{+}$we have

$$
\int_{\Omega \times \Omega} \frac{\left|u_{\lambda}(x)-u_{\lambda}(y)\right|^{p(x, y)}}{|x-y|^{N+s p(x, y)}} d x d y+\int_{\Omega}\left|u_{\lambda}\right|^{q(x)} d x=\lambda \int_{\Omega} V(x)\left|u_{\lambda}\right|^{r(x)} d x .
$$

By the definition of the set $X_{0}^{+}$, we have $\int_{\Omega} V(x)\left|u_{\lambda}\right|^{r(x)} d x>0$. By 3.22 and the definition of $\lambda_{*}$ and the fact that $\lambda<\lambda_{*}$, we deduce that

$$
\begin{aligned}
& \int_{\Omega \times \Omega} \frac{\left|u_{\lambda}(x)-u_{\lambda}(y)\right|^{p(x, y)}}{|x-y|^{N+s p(x, y)}} d x d y+\int_{\Omega}\left|u_{\lambda}\right|^{q(x)} d x \\
\geq & \lambda_{*} \int_{\Omega} V(x)\left|u_{\lambda}\right|^{r(x)} d x>\lambda \int_{\Omega} V(x)\left|u_{\lambda}\right|^{r(x)} d x \\
= & \int_{\Omega \times \Omega} \frac{\left|u_{\lambda}(x)-u_{\lambda}(y)\right|^{p(x, y)}}{|x-y|^{N+s p(x, y)}} d x d y+\int_{\Omega}\left|u_{\lambda}\right|^{q(x)} d x,
\end{aligned}
$$

which is a contradiction. Therefore, the proof of Theorem 3.3 is now completed. 


\section{Acknowledgments}

The author would like to thank the referees for their suggestions and helpful comments which improved the presentation of the original manuscript. This research is supported by Vietnam National Foundation for Science and Technology Development (NAFOSTED) (Grant N.101.02.2017.04).

\section{References}

[1] A. Abdou and A. Marcos, Existence and multiplicity of solutions for a Dirichlet problem involving perturbed $p(x)$-Laplacian operator, Electron. J. Differential Equations 2016 (2016), no. 197, 19 pp.

[2] E. Acerbi and G. Mingione, Regularity results for a class of functionals with nonstandard growth, Arch. Ration. Mech. Anal. 156 (2001), no. 2, 121-140.

[3] C. O. Alves and M. C. Ferreira, Existence of solutions for a class of $p(x)$-Laplacian equations involving a concave-convex nonlinearity with critical growth in $\mathbb{R}^{N}$, Topol. Methods Nonlinear Anal. 45 (2015), no. 2, 399-422.

[4] S. Antontsev, F. Mirandac and L. Santos, Blow-up and finite time extinction for $p(x, t)$-curl systems arising in electromagnetism, J. Math. Anal. Appl. 440 (2016), no. $1,300-322$.

[5] G. Autuori and P. Pucci, Elliptic problems involving the fractional Laplacian in $\mathbb{R}^{N}$, J. Differential Equations 255 (2013), no. 8, 2340-2362.

[6] A. Baalal and M. Berghout, Traces and fractional Sobolev extension domains with variable exponent, Int. J. Math. Anal. (Ruse) 12 (2018), no. 2, 85-98.

[7] A. Bahrouni, Comparison and sub-supersolution principles for the fractional $p(x)$ Laplacian, J. Math. Anal. Appl. 458 (2018), no. 2, 1363-1372.

[8] A. Bahrouni and V. D. Rădulescu, On a new fractional Sobolev space and applications to nonlocal variational problems with variable exponent, Discrete Contin. Dyn. Syst. Ser. S 11 (2018), no. 3, 379-389.

[9] A. Bahrouni and D. Repovš, Existence and nonexistence of solutions for $p(x)$-curl systems arising in electromagnetism, Complex Var. Elliptic Equ. 63 (2018), no. 2, $292-301$. 
[10] G. Barletta, A. Chinnì and D. O'Regan, Existence results for a Neumann problem involving the $p(x)$-Laplacian with discontinuous nonlinearities, Nonlinear Anal. Real World Appl. 27 (2016), 312-325.

[11] R. Bartolo and G. M. Bisci, Asymptotically linear fractional p-Laplacian equations, Ann. Mat. Pura Appl. (4) 196 (2017), no. 2, 427-442.

[12] Z. Binlin, G. M. Bisci and R. Servadei, Superlinear nonlocal fractional problems with infinitely many solutions, Nonlinearity 28 (2015), no. 7, 2247-2264.

[13] G. M. Bisci, V. D. Radulescu and R. Servadei, Variational Methods for Nonlocal Fractional Problems, Encyclopedia of Mathematics and its Applications 162, Cambridge University Press, Cambridge, 2016.

[14] M. Bouslimi and K. Kefi, Existence of solution for an indefinite weight quasilinear problem with variable exponent, Complex Var. Elliptic Equ. 58 (2013), no. 12, 16551666.

[15] L. Diening, P. Harjulehto, P. Hästö and M. Růžička, Lebesgue and Sobolev Spaces with Variable Exponents, Lecture Notes in Mathematics 2017, Springer, Heidelberg, 2011.

[16] D. E. Edmunds and J. Rákosník, Sobolev embeddings with variable exponent, Studia Math. 143 (2000), no. 3, 267-293.

[17] I. Ekeland, On the variational principle, J. Math. Anal. Appl. 47 (1974), 324-353.

[18] A. Fiscella, G. M. Bisci and R. Servadei, Multiplicity results for fractional Laplace problems with critical growth, Manuscripta Math. 155 (2018), no. 3-4, 369-388.

[19] U. Kaufmann, J. D. Rossi and R. Vidal, Fractional Sobolev spaces with variable exponents and fractional $p(x)$-Laplacians, Electronic J. Qual. Theory Differ. Equ. 2017 (2017), no. 76, 10 pp.

[20] K. Kefi, p(x)-Laplacian with indefinite weight, Proc. Amer. Math. Soc. 139 (2011), no. $12,4351-4360$.

[21] M. Mihăilescu and V. Rădulescu, Eigenvalue problems with weight and variable exponent for the Laplace operator, Anal. Appl. (Singap.) 8 (2010), no. 3, 235-246.

[22] _ Concentration phenomena in nonlinear eigenvalue problems with variable exponents and sign-changing potential, J. Anal. Math. 111 (2010), 267-287. 
[23] J. Musielak, Orlicz spaces and modular spaces, Lecture Notes in Mathematics 1034, Springer-Verlag, Berlin, 1983.

[24] L. M. Del Pezzo and J. D. Rossi, Traces for fractional Sobolev spaces with variable exponents, Adv. Oper. Theory 2 (2017), no. 4, 435-446.

[25] P. Pucci, M. Xiang and B. Zhang, Existence and multiplicity of entire solutions for fractional p-Kirchhoff equations, Adv. Nonlinear Anal. 5 (2016), no. 1, 27-55.

[26] V. D. Rădulescu, Nonlinear elliptic equations with variable exponent: old and new, Nonlinear Anal. 121 (2015), 336-369.

[27] M. Růžička, Electrorheological Fluids: Modeling and Mathematical Theory, Lecture Notes in Mathematics 1748, Springer-Verlag, Berlin 2000.

[28] R. Servadei and E. Valdinoci, Variational methods for non-local operators of elliptic type, Discrete Contin. Dyn. Syst. 33 (2013), no. 5, 2105-2137.

[29] M. Xiang, B. Zhang and D. Yang, Multiplicity results for variable-order fractional Laplacian equations with variable growth, Nonlinear Anal. 178 (2019), 190-204.

Nguyen Thanh Chung

Department of Mathematics, Quang Binh University, 312 Ly Thuong Kiet, Dong Hoi, Quang Binh, Vietnam

E-mail address: ntchung82@yahoo.com 